Titre : Le rôle de la perception du risque dans la réticence à la vaccination et le défi de la communication

Auteur: Conrad G. Brunk

Publication : La santé publique à une ère marquée par le doute - Origines religieuses et culturelles de l'hésitation des Canadiens face à la vaccination

Directeurs : Paul Bramadat, Maryse Guay, Julie A. Bettinger et Réal Roy

Pages : $73-99$

ISBN : 978-2-7622-0359-2

URI : http://hdl.handle.net/11143/16030

DOI : https://doi.org/10.17118/11143/16030

Éditeur : Les Éditions de l'Université de Sherbrooke (ÉDUS) 


\title{
Le rôle de la perception du risque dans la réticence à la vaccination et le défi de la communication
}

\author{
CONRAD G. BRUNK
}

Dès le début de ce projet de livre visant la réticence à la vaccination, l'équipe de chercheurs a rencontré un groupe d'experts et d'intervenants bien informées. Nous avons entendu des personnes ayant des opinions bien arrêtées, mais opposées. Deux participants ont fait des déclarations bien senties. L'une, épidémiologiste, connaissait bien les effets des maladies infectieuses sur les populations et le rôle de la vaccination pour les maîtriser et les prévenir. Mère, elle a aussi raconté comment son enfant, peu de temps après avoir reçu le vaccin RRO (rougeole, rubéole, oreillons), a commencé à éprouver des troubles du sommeil, de l'alimentation et des fonctions sensorielles; par la suite, on lui a diagnostiqué un trouble envahissant du développement. Lors de notre rencontre, elle a dit savoir qu'on n'avait pas pu établir de lien causal entre le vaccin et l'autisme et que l'étude qui en avait fait état était trompeuse. Cependant, puisque, au moins sur le plan temporel, les deux pouvaient être étroitement liés dans le cas de son enfant, la possibilité d'une relation de cause à effet devint si évidente pour elle qu'elle se sentit effroyablement coupable d'avoir décidé de le faire vacciner. Scientifique elle-même, elle reconnaissait l'ensemble des bienfaits que la vaccination apporte à la société en limitant les éclosions de maladies dangereuses, notamment chez les enfants. Malgré tout, vu le fardeau que doivent supporter quotidiennement sa famille et celle de nombreux enfants autistes, elle s'interrogeait sur la possibilité que ce fardeau trouve son origine dans sa décision de vaccination et qu'il soit trop lourd à assumer, même en tenant compte des bienfaits de celle-ci.

Lors de cette même rencontre d'équipe, nous avons aussi entendu un témoignage passionné, de la part d'une infirmière travaillant aux soins intensifs pédiatriques d'un hôpital établi dans un quartier comptant un grand nombre d'enfants non vaccinés. Selon elle, les souffrances que subissaient les enfants admis aux soins intensifs à la suite d'une maladie pouvant être prévenue par un vaccin étaient «tout à fait inacceptables ». Elle a ardemment soutenu que les professionnels de la santé avaient l'obligation morale de mieux informer leurs parents afin, disait-elle, "de leur permettre d'exercer le choix de sauver la vie de leur enfant». Pour elle, les avantages de la vaccination des enfants sont plus qu'évidents et le refus des 
parents ne peut provenir que de leur ignorance de ce fait et d'une crainte d'effets indésirables sans fondement scientifique.

Dans de nombreux chapitres de cet ouvrage, on cite également des déclarations de personnes réticentes à la vaccination. Elles proviennent autant de gens qui ont persistéà refuser la vaccination pour eux-mêmes ou pour leurs enfants, que d'autres qui l'ont acceptée malgré leurs réserves (Guay et coll., 2014). Ces témoignages font état de toutes les considérations bien connues des personnes qui s'interrogent profondément au sujet de la vaccination, à savoir la conviction que des effets secondaires graves suivront, la confiance en la science et en la motivation des scientifiques, des gouvernements, des grandes sociétés et des professionnels de la santé, l'opinion des homologues et des êtres chers, les convictions religieuses, les obligations parentales, etc. On n'a pas demandé à ces personnes de pousser leur réflexion plus loin, de sorte que nous n'avons pas de réponse à ces questions plus approfondies: quels facteurs amènent ces personnes à choisir de manière définitive les considérations qui seront décisives pour elles? Pourquoi accordent-elles plus de priorité à certains risques plutôt qu'à d'autres dans leur décision? Pour quelles raisons choisissent-elles les autorités qu'elles citent (données scientifiques, témoignage anecdotique, Internet, textes sacrés, etc.)? Pourquoi certaines d'entre elles concluent-elles que la possibilité de dommages à cause de la vaccination (bien que ce soit improbable) est plus ou moins importante que la possibilité de causer des dommages en refusant la vaccination?

Nous avons l'intention, dans ce chapitre, de faire une analyse exhaustive des hypothèses sous-jacentes pour chacun de ces points de vue si variés et apparemment conflictuels. II y a, dans ces témoignages et dans bien d'autres qui soutiennent la vaccination ou qui s'y opposent, bien plus qu'il n'y paraît. Le présent ouvrage a pour but d'expliquer et d'analyser les nombreux facteurs qui interviennent dans la controverse au sujet de la vaccination. Dans ce chapitre-ci, nous traiterons des différents moyens par lesquels la perception des risques et leur acceptabilité exercent une influence sur la position que veulent s'arroger les antagonistes dans les débats. Les témoignages des deux professionnelles citées au début du chapitre dressent le portrait des risques et des bienfaits de la vaccination. L'une d'entre elles estime qu'avoir pris ce risque fut une erreur moralement blâmable; l'autre est d'avis que le défaut de prévenir le risque de la non-vaccination, chez les enfants, est également blâmable. Ces deux femmes sont des professionnelles et des scientifiques accomplies. La mésentente entre elles ne provient pas de compréhensions différentes des données scientifiques ellesmêmes, mais de la manière de les interpréter et de les transformer en un geste raisonnable. Notre but est de mieux comprendre ce processus de transformation. Comment les gens en arrivent-ils à des conclusions sur ce qui rend le risque de vaccination acceptable? Quand nous serons en mesure de cerner les facteurs non scientifiques supplémentaires qui entrent dans la formulation de conclusions individuelles sur l'innocuité d'un vaccin, nous serons aptes à mieux comprendre les enjeux et à donner une réponse plus efficace aux inquiétudes.

Les autres chapitres du présent ouvrage décrivent les nombreux facteurs ayant une incidence sur les attitudes du public, en ce qui concerne l'acceptation et le refus de la vaccination. Parmi eux figurent les suivants: l'ambivalence d'une partie de la population, vivant sous le système de médecine allopathique dominant en Occident, mais attirée par les thérapies alternatives; la crainte des " grands », notamment les grands de la fabrication des médicaments et les gouvernements, qui seraient une menace pour l'autonomie et la responsabilité individuelle (ou parentale); les convictions et pratiques inspirées de la religion 
ou de la «culture », qui exercent souvent une influence critique sur la réflexion des gens en matière de santé.

Plusieurs auteurs ont aussi cerné un autre facteur, souvent appelé l'« analphabétisme scientifique ", qui fait l'objet de discussions entre les scientifiques, les professionnels de la santé et des experts d'autres domaines. Bien qu'on en fasse souvent l'explication à l'échelle mondiale de ce que les experts considèrent comme une compréhension irrationnelle des risques et des bienfaits de la vaccination, cette expression tend à réunir plusieurs aspects distincts de la question relative au risque acceptable. Le risque acceptable est un concept complexe. Il fait surgir des questions dont certaines peuvent obtenir réponse grâce à la technologie, soutenue par la science. D'autres cependant échappent totalement à la science, parce qu'il ne s'agit pas de questions scientifiques.

Il y a au moins trois manières différentes de considérer comme " non scientifiques »les choix que des personnes exercent :

1. Il se peut que les gens comprennent mal les fondements scientifiques d'interventions médicales telles que la vaccination et ne puissent pas en saisir toute l'efficacité dans la prévention de la maladie et son soulagement; il est aussi possible que l'on croie aux répercussions négatives des vaccins sur la santé, même si cela n'a pas été validé par des données scientifiques.

2. Certaines gens ont tendance à faire des inférences erronées au sujet de la probabilité des risques et des bienfaits de la vaccination, simplement parce qu'elles ne connaissent pas les probabilités et la statistique.

3. Certaines personnes peuvent prendre des décisions inappropriées au sujet de l'acceptabilité des risques et des bienfaits, parce qu'elles contestent les approches bien reconnues en matière de décisions rationnelles.

On doit tout de même accepter qu'il existe une certaine dépendance entre ces faits reconnus et ces hypothèses et les questions fort différentes qui en découlent. L'ignorance des fondements scientifiques de la vaccination et la confiance accordée aux témoignages anecdotiques font souvent surgir des craintes irraisonnées de répercussions indésirables et mènent à croire fermement que la vaccination est la cause d'une maladie ou d'un handicap ou à surestimer de manière flagrante la probabilité de telles répercussions. La méconnaissance de la science peut aussi se traduire par une sous-estimation des bienfaits des programmes de vaccination, pourtant connus, voire au déni total de leur existence. Les médias populaires publient sans arrêt des allégations appuyées sur des données pseudo-scientifiques au sujet des risques et des bienfaits de ces programmes et les profanes n'ont souvent pas à leur disposition les ressources nécessaires pour en faire une évaluation critique. Par la suite, ils en tirent souvent des conclusions au sujet de la probabilité relative des risques et des bienfaits de la vaccination qui vont clairement à l'encontre des preuves scientifiques. Cette façon de faire peut les mener à prendre des décisions de refus pour eux-mêmes ou pour leurs enfants et à affirmer que la vaccination n'est dans l'intérêt de personne; ils peuvent même la juger contraire à l'éthique.

La documentation concernant les aspects psychologiques de la prise de décisions contient une foule d'études sur le mauvais jugement dont la plupart d'entre nous faisons preuve quand il est question de probabilités (Kahneman, 2011). II n'est pas toujours facile de transformer une probabilité statistique en décision pratique, même pour un expert. Les profanes sont encore plus enclins à errer dans l'évaluation des probabilités. Il arrive souvent qu'une même probabilité statistique soit vue différemment, selon la manière dont on l'a exprimée. La plupart des gens, par exemple, verront une différence entre la probabilité de sauver huit vies sur dix 
et celle de perdre deux vies sur dix, cette dernière étant la pire (Kahneman, 2011; Kahneman et coll., 1982; Tversky et Kahneman, 1974). Ces faits concernant la psychologie humaine sont à l'origine des préjugés qu'ont les gens sur l'efficacité (et l'acceptabilité) de la vaccination. On accordera plus d'importance au fait que le risque connu de répercussions indésirables touche 15 personnes vaccinées sur 10000 qu'à celui que les 9985 personnes restantes tireront du vaccin un bienfait probable qui pourrait même leur sauver la vie.

Il est donc évident que la troisième allégation ci-dessus ne se rapporte pas réellement à l'« analphabétisme scientifique », puisque les questions qu'elle vise ne relèvent pas du domaine de la science. II ne s'agit pas de contester l'ampleur des risques et des bienfaits (ce qui est en grande partie d'ordre scientifique), mais bien de l'acceptabilité comparative des risques et des bienfaits de la vaccination. L'acceptabilité du risque (qui correspond à la définition technique de l'« innocuité ») repose clairement sur un jugement de valeur et non pas sur l'évaluation ou l'estimation scientifique du risque (Rescher, 1983). Cela mène toujours à la question : «En vertu des valeurs de quel intervenant le risque est-il jugé acceptable? » En effet, les gens ont recours à des valeurs différentes au moment d'envisager l'acceptabilité du risque. Certaines personnes ont une tolérance très grande à l'égard du risque (ce sont les «preneurs de risque »). Pour les adeptes des sports extrêmes, par exemple, le risque est recherché comme une valeur en soi; ce groupe rassemble les «téméraires ». Pour les autres (sans doute la majorité d'entre nous), le degré de risque à tolérer est beaucoup plus faible; on appelle ce groupe les «craintifs ». En vérité, cependant, toute cette question est plus complexe que ne peut l'expliquer une simple typologie, car nous savons tous que certains risques sont acceptables pour une personne et d'autres moins et que cela dépend d'une multitude d'autres enjeux qui se réunissent dans leur mode de perception. Nous sommes nombreux à connaître des personnes qui ne mangent que des aliments biologiques (parfois parce qu'elles ne tolèrent pas le risque associé aux pesticides) ou qui ont peur de l'avion (l'idée de mourir dans un écrasement les effraie), mais qui font du ski ou sont de grands fumeurs (peut-être veulent-ils circonscrire d'autres risques, étant prêts à en prendre de plus grands, comme s'il s'agissait d'une accoutumance).

Le principal sujet de ce chapitre porte sur notre capacité à juger l'acceptabilité d'un risque. II semble que ce volet de la controverse sociale au sujet du risque environnemental et du risque pour la santé humaine (des changements climatiques aux plantes et animaux génétiquement modifiés, en passant par les transfusions sanguines et la vaccination) soit le moins bien compris par les experts du risque. On en a pour preuve la caractérisation de certains jugements sur l'acceptabilité du risque comme étant une démonstration de l'« analphabétisme scientifique ». C'est pourquoi, lorsqu'il s'agit de promouvoir les politiques et les projets qui traitent du problème du risque inacceptable et sont destinés aux opposants ou aux réticents à une pratique médicale reconnue, les experts éprouvent souvent un échec total dans leurs efforts de communication. Mon objectif, dans les pages qui suivent, consiste à expliquer les aspects non scientifiques des attitudes d'un public qui perçoit des risques dans la technologie en général et, en conséquence et plus précisément, dans la vaccination. Une juste compréhension de tous les facteurs qui influent sur l'acceptation ou l'hésitation à l'égard de la vaccination est essentielle, à quelque partie que l'on appartienne, pour mieux saisir la position de son opposant et communiquer efficacement avec lui. 
Les professionnels de la santé, les gouvernements et les autorités de santé publique font la promotion des programmes de vaccination parce qu'il existe un solide consensus entre les scientifiques, les fabricants de vaccins et les praticiens de la santé indiquant que ces programmes sont un bon outil de prévention, voire d'élimination de maladies dangereuses pour l'être humain. Comme le démontrent Bettinger et MacDonald (au chapitre 8, plus loin), une participation générale de la population est essentielle à l'efficacité de la plupart des programmes de vaccination. Un relativement petit nombre de non-vaccinés, dans une population à risque, peut rendre un programme moins efficace en permettant la survie de réservoirs de la maladie, qui constituera alors une menace constante. Si cela découle d'une réticence active dans des sous-populations ayant une opinion divergente sur la valeur ou l'acceptabilité d'un programme de vaccination, les responsables de la santé et les praticiens se sentiront naturellement obligés d'amener ces groupes à accepter le programme et à y participer. L'approche typique est fondée sur une hypothèse, à savoir que les réticents et les opposants ne comprennent tout simplement pas les notions scientifiques soutenant l'efficacité des programmes; dès lors, on pense que le moyen de transformer cette attitude consiste à mieux propager ces notions.

De toute évidence, un des facteurs les plus importants, en ce qui concerne l'acceptation ou la réticence à la vaccination, est la compréhension et l'acceptation des données scientifiques médicales. La situation n'est pas seulement le résultat de l'ignorance ou de l'analphabétisme scientifique; il y a aussi, souvent, une méfiance à l'égard de cette science, si bien comprise soitelle. De nombreux motifs encouragent la méfiance à l'égard de la science et de la pratique médicale. En voici quelques exemples:

- Une méfiance générale à l'égard de la science, qui découle de reportages sur des études scientifiques apparemment conflictuels;

- Les répercussions d'accidents ou de maladies ayant été causées par des technologies ou des procédures que les experts scientifiques avaient déclarées «sans danger» (la thalidomide, le Vioxx, etc.);

- Des études pseudoscientifiques (par exemple, l'étude Wakefield de 1998 qui associait le vaccin RRO à l'autisme et qui fut discréditée par la suite; voir Wakefield et coll., 1998; Editors of The Lancet, 2010) qui allèguent que la vaccination et d'autres procédures médicales comportent de grands risques;

- Des incertitudes réelles provenant de nouvelles données scientifiques causant une controverse dans les milieux de la science;

- Un rejet des paradigmes dominants de la science et de la pratique médicale au profit de pratiques et de philosophies « alternatives » (naturopathie, médecine traditionnelle, homéopathie, etc.);

- L'importance grandissante de ce que l'on appelle le « principe de précaution », c'est-à-dire le concept qui constitue, pour bien des gens, le fondement logique d'une position prudente en ce qui a trait à l'utilisation des nouvelles technologies, lorsqu'on perçoit des incertitudes importantes à l'échelle des données sous-jacentes. 
Dans la société contemporaine, on ne manque pas d'exemples de méfiance à l'égard des protocoles scientifiques utilisés pour la mise au point et l'administration des vaccins dans le cadre de la gestion de la maladie. Cette "crise de la confiance ", dont traite Bramadat au chapitre 1, se manifeste dans toute la société canadienne et s'est répandue encore plus facilement grâce aux rumeurs circulant sur Internet et les médias sociaux. Elle a une incidence sur la manière de penser individuelle, même chez les personnes assez bien renseignées en matière de sciences, et elle réunit des gens qui partagent le même avis. En fait, des études (Frewer, Shepherd et Sparks, 1994) ont démontré que, dans de nombreuses controverses ayant cours dans notre société (aliments génétiquement modifiés, production d'énergie nucléaire, radiations provenant des téléphones cellulaires, et ainsi de suite), les sous-populations qui rejettent les évaluations dominantes des experts du risque ne sont généralement pas moins et souvent plus informées en matière scientifique que le reste de la population. Ces études remettent donc en cause, et profondément, l'adoption généralement répandue du « modèle du déficit des connaissances », selon lequel l'acceptation d'un risque divergent est un signe d'analphabétisme scientifique (que l'on pourrait évidemment corriger par l'éducation). Toutefois, quand on analyse les controverses à l'aide de ce modèle, ce dernier ne saisit pas les nombreux facteurs qui entrent en jeu dans le phénomène de réticence à la vaccination au sein de notre société.

Avant d'examiner ces facteurs, je rappelle un de mes principaux arguments, à savoir que nos observations ne permettent souvent pas de saisir que les gens étudient les probabilités de risques et de bienfaits pour en tirer non seulement un compromis rationnel et statistique entre ces deux volets de la question, mais aussi des conclusions dans lesquelles sont imbriquées de complexes jugements de valeur. On notera par ailleurs que, dans ce chapitre, le mot « valeur » désigne toutes les considérations qui nous permettent de classer tout élément comme bon ou mauvais, exact ou erroné ou valable ou sans importance. Les valeurs correspondent à nos préférences pour ce que nous expérimentons. Selon la définition conventionnelle, les valeurs sont le contraire des "faits». Les faits sont des choses et des circonstances observables dans l'univers. Un fait « est ». Une valeur, par contre, représente une attitude envers ce que l'on connaît ou connaîtra. Nos valeurs nous proviennent de nombreuses sources. Certaines nous sont innées, tandis que d'autres nous viennent de nos parents, de nos amis et de notre culture, tout au long de notre vie. Les sociétés et les cultures s'imprègnent aussi de valeurs. Dans l'histoire, la religion est l'une des plus importantes sources de valeurs culturelles et personnelles. La vision du monde qui sous-tend une religion est constituée de jugements implicites et explicites au sujet des éléments signifiants ou insignifiants, acceptables ou non, et ainsi de suite. En vérité, comme de nombreuses religions mettent l'accent sur des éléments que les gens jugent très importants (v. Tillich, 1957) dans leur vie, elles préconisent des valeurs qui ne sont pas "négociables ». Parmi les groupes religieux qui refusent la vaccination, certains croient que celle-ci contrevient à une interdiction divine très claire, dont ils trouvent l'origine dans l'interprétation des écrits traditionnels. La plupart des valeurs, y compris les religieuses, n'appartiennent pas à la catégorie de celles qui ne souffrent aucun compromis. Les populations et les cultures reconnaissent, dans l'ensemble, que les valeurs sont souvent conflictuelles et qu'il faut exercer des choix. Ces choix sont souvent difficiles quand il faut déterminer si le risque vaut la peine d'être pris pour obtenir des bienfaits éventuels. 


\section{Les facteurs dans la perception du risque}

Le concept du risque, la science de l'évaluation du risque et la gestion du risque sont devenus des éléments centraux de la gestion des problèmes de protection de l'environnement et de santé dans les sociétés modernes. Le " paradigme du risque » semble intéressant, car il promet de fournir un outil objectif et rationnel pour les décisions de politiques publiques, qui permettrait d'équilibrer les avantages et les inconvénients de l'activité humaine pour la société, notamment dans le domaine technologique. Les avantages et les inconvénients, dans ce paradigme, sont des exemples de ce que l'on appelle des «valeurs». Ce que nous entendons par là ne nous vient pas uniquement de l'observation de cas empiriques et ce ne sont pas uniquement des faits concernant l'expérience humaine, mais autre chose aussi. Ce sont des jugements de valeur que nous exprimons au sujet de l'incidence de ces faits dans notre vie et qui entrent en jeu lorsqu'on discute, par exemple, de la manière de mesurer les dommages infligés par une maladie en particulier. Devrait-on établir le coût économique pour la société ou uniquement pour la personne malade? Devrait-on tenir compte du degré de souffrance subie ou faire le calcul en fonction de l'AVAQ (année de vie ajustée en fonction de la qualité), qui tient compte de la douleur et de la souffrance pour un ensemble de personnes?

Ce concept du risque est clairement fondé sur les valeurs et son évaluation, mais les sociétés pluralistes de type libéral sont fortement intéressées à appuyer les politiques publiques, dans la mesure du possible, sur une appréciation «scientifique » du risque, fondée non pas sur des valeurs, mais sur les faits. Quand cela est possible, on peut affirmer que la politique publique est fondée sur des considérations dépourvues de jugement de valeur, ce qui, en théorie, la rendrait acceptable pour toutes les personnes raisonnables, indépendamment de valeurs propres (perspectives religieuses, convictions culturelles, etc.). Évidemment, il ne faudra pas s'étonner que les membres de collectivités ayant de solides valeurs, comme les groupes religieux, se méfient de ces politiques dépourvues de jugement de valeur et les soupçonnent de cacher des valeurs qui s'opposent aux leurs. Ils ont souvent raison. Malgré tout, notre société fait grandement confiance à la science de l'évaluation du risque pour exécuter la tâche, dépendant en grande partie, mais non entièrement de données empiriques, qui consiste à établir quels seront les effets, positifs ou négatifs, de nos gestes et de notre recours aux nouvelles technologies?.

La science de l'évaluation du risque est fondée sur l'idée qu'on peut mesurer l'ampleur d'un risque à l'aide d'une formule mathématique regroupant des facteurs quantifiables l'importance des dommages (ou bienfaits) multipliée par leur probabilité de réalisation. En supposant que nous ayons résolu le problème (basé sur les valeurs) de déterminer comment quantifier et mesurer les inconvénients et les bienfaits (ce sur quoi la controverse se poursuit entre philosophes et économistes), nous pouvons mesurer les résultats et leur attribuer

1. Les régimes de réglementation de la santé et de la protection de l'environnement du Canada, des États-Unis et de la plupart des pays du G20 en sont un bon exemple. Ces pays obéissent aux règles d'accords internationaux (ceux de l'OMC, de la FAO, de l'OIE, etc.) et fondent leur système d'approbation des aliments, médicaments, produits thérapeutiques et autres sur des facteurs de risque exclusivement scientifiques, ne tenant aucun compte de considérations sociales, culturelles, éthiques ou religieuses. Plus précisément encore, l'Agence canadienne d'inspection des aliments et Santé Canada ne peuvent, en raison de leur engagement en faveur d'une réglementation basée sur la science, fonder l'analyse d'une demande d'introduction d'animaux génétiquement modifiés dans le marché canadien sur le fait que la plupart des Canadiens jugent ces animaux acceptables après examen de considérations relatives à leur bien-être (Brunk et Hartley, 2012). 
une probabilité statistique de réalisation selon les circonstances ${ }^{2}$. Dans la documentation traitant du risque, il est défini ainsi : le degré de risque est un coefficient établi en fonction des dommages totaux que peut entraîner un événement et de sa probabilité de réalisation (Rescher, 1983; Thompson, 1986).

C'est sur ce paradigme du risque qu'est fondée la pratique médicale contemporaine, sous pratiquement tous ses angles - notamment l'évaluation des nouveaux médicaments et thérapies, nouveaux vaccins inclus, qui doivent recevoir l'aval des organismes de réglementation pour être mis en marché. La « médecine fondée sur des données probantes ", devenue chez nous un mantra du secteur de la santé, se fie également à cette méthode pour évaluer l'efficacité des traitements et des interventions.

Cette approche positionne l'évaluation de ces interventions sur une base scientifique éprouvée. Nul ne doute que les soins de santé doivent être fondés, dans la mesure du possible, sur les meilleures données probantes et que la démarche d'évaluation du risque soit le meilleur moyen d'y parvenir, même si celle-ci se base inéluctablement sur certaines hypothèses non scientifiques (par exemple quand il s'agit de définir et de mesurer les dommages) ${ }^{3}$. Cependant, même avec l'aide de cette manière de mesurer le degré de risque (et de bienfaits) des interventions thérapeutiques, un problème demeure, à savoir comment déterminer jusqu'à quel degré le risque est acceptable. Dans le paradigme du risque, c'est ce qu'on appelle le problème de la gestion du risque ou de l'« évaluation de l'innocuité ». Ce problème, selon l'opinion générale, est une question de valeur, contrairement au processus d'évaluation du risque. Il est rare que l'innocuité ait pour objectif d'éliminer totalement le risque. Dans la vie courante, en effet, il n'y a guère d'activités ou de technologies qui soient sans risque. La question de savoir si une activité est sûre consiste donc, la plupart du temps, à déterminer si son degré de risque est acceptable (compte tenu des bienfaits prévus).

II est impossible de répondre à cette question, à moins d'en poser plusieurs autres : pour qui ce degré de risque est-il acceptable? Et quels sont les critères sur lesquels baser cette évaluation, le cas échéant? La science empirique ne peut y donner de réponse, car ce ne sont pas des questions scientifiques. Les évaluations sont clairement rattachées à des préférences distinctives, ainsi qu'à des cadres moraux, religieux et philosophiques différents. Les gens, en nombre croissant, trouvent inaceptable de s'exposer auxrisques bien connus et prouvés scientifiquement de la conduite automobile sans attacher sa ceinture de sécurité. Cependant, certains continuent de ne pas porter leur ceinture bien qu'ils connaissent ces données qui prouvent qu'ils risquent des blessures plus graves et même le décès dans un accident, mais ils persistent. On doit supposer qu'ils préfèrent être libérés de l'inconvénient d'une ceinture de sécurité.

La tendance chez les experts, particulièrement ceux qui veulent que toutes les décisions de politique publique soient fondées sur la science, consiste à rechercher une formule objective et rationnelle permettant d'apporter une réponse normative à la question du risque acceptable. Les possibilités les plus intéressantes sont celles qui permettent une appréciation

2. Évidemment, l'hypothèse selon laquelle les valeurs n'entrent pas en jeu dans ces questions est loin d'être vérifiée. De nombreux analystes ont démontré qu'il est illogique de faire de l'évaluation des risques une science empirique faisant abstraction des valeurs, les considérations normatives ayant leur place dans pratiquement chacun des aspects de l'analyse. Cette question échappe cependant à la portée du présent ouvrage. Le lecteur qui désire en savoir plus peut se reporter à Brunk, Haworth et Lee (1991).

3. Ibid. 
uniquement quantitative, car elles confèrent une précision quasi mathématique à la réponse. Parmi elles figurent les approches économiques typiques, qui cherchent à équilibrer les possibilités de dommages et de bienfaits (analyse risques-avantages), à les optimiser d'une façon ou d'une autre (optimalité de Pareto) ou à atteindre la plus grande réduction du risque contre le plus faible coût (coût-efficacité) (Thompson, 1986; Sen, 1985).

Ces types d'approches quantitatives conviennent très bien à de nombreux contextes, notamment lorsque la question porte sur les préférences d'un groupe seulement (évaluation du risque financier pour une personne ou une entreprise, par exemple). II existe cependant bien d'autres manières par lesquelles les populations et les groupes arrivent à une décision sur l'acceptabilité d'un risque. Ces approches ne sont pas basées sur un raisonnement quantitatif comme les précédentes. Leur rationalité s'établit par comparaison à des cadres théoriques différents, basés sur des principes éthiques, religieux ou autres. Les experts qui affectionnent des formules quantitatives pour la détermination de l'acceptabilité d'un risque qualifient souvent de «non scientifiques » ou d'« irrationnels » ceux qui utilisent des règles de prise de décision plus qualitatives ou des arguments heuristiques.

Et quels sont ces cadres utilisant des valeurs plus qualitatives qui permettent à nombre de gens d'appréhender l'acceptabilité d'un risque? Ils ont été mis au jour par de nombreuses études sur la perception du risque, dans lesquelles on cherchait à comprendre les facteurs qui mènent des profanes à des conclusions fort différentes sur l'acceptabilité d'un risque, par rapport à ce que proposent les approches quantitatives standard (Slovic, 1987; Wildavsky et Dake, 1990; Fischhoff, 1998; Fischhoff, Slovic et Lichtenstein, 2000). Les spécialistes de la discipline s'intéresseront particulièrement aux facteurs psychologiques qui pourraient mener le cerveau humain à des conclusions « irrationnelles » sur l'acceptabilité d'un risque l'irrationalité étant déterminée par le fait que les conclusions s'éloignent des algorithmes quantitatifs. Examinons brièvement les facteurs qualitatifs influençant le plus couramment la perception de l'acceptabilité d'un risque chez la plupart des individus, en portant attention aux valeurs sous-jacentes qui entrent en jeu et à la pertinence de ces facteurs dans le cheminement que suivent bien des gens désirant évaluer l'acceptabilité de la vaccination, pour eux-mêmes ou pour leurs enfants.

\section{La nouveauté du risque}

L'être humain, comme la plupart des autres animaux, a prospéré au cours des précédents millénaires parce qu'il a appris à composer avec les nombreux périls qui le guettent dans son milieu de vie. Nous avons conçu des stratégies pour répondre aux menaces courantes dans notre expérience collective, de sorte à en être moins effrayés. Devant des dangers potentiels inconnus, nous nous montrons naturellement plus prudents, jusqu'à ce que nous acquérions plus d'expérience et déterminions s'ils sont bénins ou non. II n'est donc pas étonnant que les études psychométriques concluent régulièrement que l'individu moyen accepte moins bien les risques nouveaux et inconnus que ceux qu'il connaît mieux, même si ces derniers présentent des dangers encore plus grands.

4. Ces facteurs psychologiques sont cependant reconnus comme des tendances s'étant développées dans le cerveau humain, car elles se sont très bien adaptées à l'évolution de l'espèce (Kahneman, 2011). 
Ce phénomène apparaît répétitivement chez les sujets réticents à la vaccination, selon plusieurs études (Guay et coll., 2014; Dubé et coll., 2015) dans lesquelles ces personnes ont dit préférer que leurs enfants développent une immunisation contre la maladie en y étant « exposés naturellement », plutôt que de les soumettre à la vaccination, un moyen artificiel et mal connu. Ce sentiment ressort des déclarations suivantes:

Oui, oui, je le répète, pour développer un système immunitaire, il faut attraper certaines maladies; par la suite, vous devenez plus fort et vous développez des anticorps... tout le monde ne meurt pas de la rougeole et j'ai lu que tous les enfants devraient l'attraper, parce qu'elle permet de renforcer le système immunitaire. Évidemment, il peut y avoir des complications, parfois fatales. (Dubé et coll., 2015)

Je n'ai pas vu l'utilité du vaccin (contre la varicelle), car tous les enfants de ma génération ont attrapé cette maladie. (Guay et coll., 2014)

Je crois que notre système immunitaire existe déjà et qu'il devrait normalement suffire; autrefois, les enfants survivaient, même sans vaccin. (Guay et coll., 2014)

Pour de nombreux experts du risque (von Winterfeldt, 1992; Tversky et Kahneman, 1974), cette tendance humaine est « irrationnelle » à bien des points de vue. Elle leur semble comporter plusieurs erreurs de jugement. La première consiste à surestimer l'ampleur ou la probabilité du risque inconnu, sans que cela soit appuyé par les données probantes disponibles. Ce point de vue serait illustré dans la récente controverse faisant rage en Colombie-Britannique et ailleurs au sujet de l'installation de compteurs «intelligents » pour mesurer la consommation d'électricité des ménages. Selon une évaluation scientifique, cette technologie ne serait guère dangereuse pour la santé humaine, mais sa nouveauté a fait en sorte que les gens ont surestimé le risque. La deuxième erreur consiste à trouver un risque inacceptable même s'il est faible comparativement aux bienfaits à prévoir. Un nouveau vaccin, par exemple, engendrera des craintes, alors même que ses bienfaits sont considérables et bien démontrés.

Ces jugements seront jugés irrationnels uniquement s'il y a suffisamment de preuves scientifiques pour démontrer que le risque inconnu est en fait très faible et qu'il n'y a aucune autre raison de le trouver inacceptable. Dans bien des discussions sur le risque, il se peut que ces suppositions, prises individuellement ou collectivement, ne soient pas justifiées. Autrement dit, la connaissance scientifique du risque inconnu ou nouveau peut être insuffisante, auquel cas son ampleur réelle est incertaine. Une aversion prudente, pour ce risque potentiel, pourrait donc être appropriée. Il pourrait être bien plus grand que les données disponibles ne l'indiquent. Il peut aussi y avoir d'autres facteurs à considérer au sujet de l'acceptabilité du risque, comme ceux qui sont indiqués ci-dessous. Parfois, les experts ou ceux qui ont grandement à gagner à commercialiser un nouveau produit amplifient le degré de certitude scientifique au sujet des risques potentiels ou évitent d'étaler quelque manque de connaissances. On en a pour preuve l'exemple du Vioxx, un médicament contre l'arthrite, qui a entraîné des milliers d'accidents cardiovasculaires, y compris des infarctus.

Comme indiqué précédemment, une inquiétude au sujet d'un nouveau vaccin témoigne de cette aversion naturelle envers les risques inconnus (et donc souvent incertains). Ceux qui sont réticents à la vaccination, pour eux-mêmes ou pour leurs êtres chers, croient souvent (nonobstant que ce soit sur de faibles bases scientifiques) qu'un vaccin peut entraîner une maladie ou un syndrome redoutable. Si, comme dans le cas de l'autisme, les causes sousjacentes sont elles-mêmes fort incertaines ou contestées, l'homme aura naturellement 
tendance à se montrer prudent devant chacune d'entre elles. Il prendra au sérieux toute suggestion voulant que le vaccin en soit une cause possible et sa tendance naturelle à l'aversion entrera en jeu. Combinée à d'autres facteurs tels ceux qui sont décrits ci-dessous, elle s'en trouvera grandement renforcée.

Les études psychométriques renvoient souvent à un phénomène appelé le « facteur d'épouvante » (Slovic, 1987). Il est question ici du fait que les torts, les maladies (pour la plupart) et les manières de mourir ne sont pas tous évalués de la même façon par tous les individus. Ils ne sont certainement pas évalués comme le font les formules usuelles de détermination du risque, soit d'après un dénominateur commun (tel que le coût pour l'économie). L'individu moyen n'évalue pas le risque de mourir de cette façon, indépendamment de la forme que ce décès prendra. Certaines manières de mourir inspirent plus de crainte que d'autres (mort dans un écrasement d'avion ou dans un accident de voiture). Certaines maladies sont plus effrayantes que d'autres (par exemple le cancer par rapport aux maladies cardiaques). De telles différences de perception ne s'expliquent pas en recourant à un critère commun (par exemple le degré de douleur provoqué selon les options, la probabilité de décès ou la fréquence) (Rescher, 1983; Thompson, 1986). Les sociologues et les psychologues seraient peut-être en mesure d'expliquer pourquoi les gens acquièrent leurs préférences, mais il n'existe pas de conception convenue de la rationalité grâce à laquelle on pourrait dire qu'une préférence pour telle manière de mourir est supérieure à une autre. Ce sont des préférences, tout simplement.

Les fausses allégations qui circulent librement dans la sous-culture anti-vaccination (par exemple que des affections dangereuses et mal comprises comme l'autisme et différentes maladies autoimmunes sont associées aux vaccins chez l'enfant) montrent la puissance du facteur d'épouvante dans la perception du risque. Dans notre société, l'autisme est clairement qualifié de syndrome redoutable, comme le démontre le témoignage maternel cité au début du chapitre. Comment une personne peut-elle justifier par la raison le refus d'une pratique médicale largement reconnue, qui promet de prévenir avec une grande certitude un risque grave (associé à la rubéole, la diphtérie, etc.), au profit d'une pratique (la non-vaccination) qui est conçue pour la protection individuelle ou celle des êtres chers contre des effets indésirables imaginaires? Pour répondre à cette question, il faut entre autres choses se dire que les maladies prévenues par un vaccin, quoique gravement débilitantes et même mortelles dans bien des cas, sont des maladies que le genre humain endure depuis des siècles, quand il n'en meurt pas, et que leur étiologie et leur impact sur l'homme sont bien connus. On peut donc dire que ce sont des « institutions » de l'expérience humaine, dont l'institutionnalisation est renforcée par le fait que, grâce à l'expansion de la vaccination, ces maladies sont généralement aujourd'hui inconnues, comme l'expliquent Bettinger et MacDonald dans le huitième chapitre. Pour l'individu moyen, ce ne sont guère que des abstractions, des idées, des anecdotes. Quand on compare les risques très certains et probables de maladies bien connues et peu redoutables (comme la rougeole, les oreillons et la rubéole) aux risques très incertains et improbables des effets indésirables moins connus et plus redoutables - même entièrement imaginaires - de la vaccination contre ces maladies, dont l'autisme, il est parfois difficile de prévoir le résultat sur la décision de vaccination ou de non-vaccination. Ajoutons à cela qu'une grande méfiance à l'égard des professions scientifiques et médicales, une perception que le choix est imposé par des agents puissants (auxquels on n'accorde pas confiance) (v. Bramadat, au chapitre 1, concernant la crise de confiance et de vérité) et la forte crainte d'être à l'origine de la souffrance d'une autre personne (surtout un enfant) peuvent aboutir à une décision de non-vaccination. 


\section{Répartition des risques et des bienfaits}

Un autre facteur important exerce une influence sur l'acceptabilité dans le traitement psychologique de la perception des risques; l'individu doit en effet estimer si les bienfaits (ou la réduction du risque) de la nouvelle technologie se trouvent répartis de manière équitable et juste. On ne peut en effet pas toujours affirmer que les bienfaits d'une technologie ou d'une pratique agissent au profit des personnes qui sont exposées au risque. C'est d'ailleurs ce qui se produit dans le cas de nombreuses technologies, sinon de la plupart d'entre elles. Ceux qui profitent de l'électricité générée par une centrale nucléaire ne sont habituellement pas ceux qui seront exposés aux radiations lorsqu'ils travaillent à l'intérieur de l'installation ou vivent à proximité du lieu de stockage des déchets radioactifs. Ceux qui profitent de l'exportation du pétrole ne sont sans doute pas ceux qui tirent leur subsistance des eaux sillonnées par les navires pétroliers ou des terres que traversent les oléoducs.

Dans les sociétés modernes, la quasi-totalité des citoyens partage le même principe moral fondamental, à savoir qu'aucune personne ni aucun groupe ne doit être avantagé aux dépens d'autrui. En fait, ce principe est sans doute la valeur morale qui est la plus répandue et la moins contestée dans les sociétés démocratiques libérales et c'est sur lui qu'est bâti le droit. Si distinctes que soient les populations sous l'angle de leur système global de valeurs, de religion ou de philosophie, elles s'entendront probablement sur le principe suivant: " La liberté de l'un, dans la recherche de son propre intérêt, est limitée par le droit des autres à ne pas en subir de dommages » (Mill, 1859). En revanche, si répandu que soit ce principe, il est étonnant de voir combien de fois il est ignoré par les experts de la gestion du risque, selon qui, en général, la seule question à se poser au sujet de l'acceptabilité du risque consiste à établir si la somme des risques (pour toutes les personnes touchées) est supérieure à la somme des bienfaits (pour tous les bénéficiaires). Dans l'affirmative, le risque est déclaré acceptable. Par contre, si les personnes qui doivent subir un dommage ne sont pas celles qui profiteront des bienfaits, comme dans l'exemple de la centrale nucléaire, les premières considéreront probablement la situation comme inéquitable, au profit des bénéficiaires. En ce qui concerne les questions d'innocuité, cette approche réduite à l'équilibre entre les risques et les bienfaits est logique pour les bénéficiaires, mais beaucoup moins pour ceux qui supportent le risque - et ils n'ont pas tort. Pourquoi devraient-ils tolérer des risques supplémentaires, si négligeables soient-ils, si les bienfaits vont à d'autres?

La solution à ce problème fait partie des difficiles objectifs de la théorie politique et économique. La politique publique peine à y répondre, car, la plupart du temps et inéluctablement, elle favorise certains intérêts plutôt que d'autres. Malgré tout, aucun effort d'imagination d'ordre moral ou scientifique n'est nécessaire pour comprendre pourquoi certaines personnes, pressentant qu'on leur impose des risques pour que d'autres en recueillent les bienfaits (même si, dit-on, cela entraîne une réduction du risque pour ellesmêmes), en viendront à la conclusion que ces risques, quelle que soit leur ampleur, sont tout à fait inacceptables. Tout individu ayant l'impression d'être injustement mis en danger ne verra probablement pas le risque comme étant acceptable et n'accordera aucune valeur à l'ampleur ou à la probabilité de dommages établies par des scientifiques. 
Cette perception du risque se reflète clairement dans le phénomène de réticence à la vaccination, dans nos sociétés. Les professionnels de la santé font parfois la promotion des programmes de vaccination en les présentant comme des éléments essentiels à la santé de la population, dans le but de réduire l'incidence d'une maladie grave dans la population en général. Pour atteindre cet objectif, il faut vacciner la plus grande partie possible de la population vulnérable, puisque le refus de certaines personnes, même en petit nombre, risque de nuire à l'efficacité du programme. Dans cette situation, plusieurs verront la vaccination, pour eux-mêmes ou pour leurs enfants, comme une méthode qui profitera sans doute plus à la population en général qu'à eux-mêmes et cela particulièrement si ces personnes (voir la section précédente) estiment qu'elle leur impose des risques justifiés par des bienfaits (réduction des risques comprise) allant à autrui.

Les professionnels de la santé (et bien des gens ayant à cœur l'intérêt public) considéreront cette attitude comme antisociale et individualiste, car elle accorde la priorité aux droits ou intérêts individuels plutôt qu'à ceux de la société. Cet individualisme endurci est fortement enraciné dans la culture et la politique libertarienne de l'Amérique du Nord, notamment aux États-Unis. Plus on est convaincu des concepts du libertarisme, plus naturellement on sera disposé à adopter cette approche dans l'examen de l'acceptabilité du risque de vaccination (Petts et Niemeyer, 2004).

\section{Le risque pris délibérément ou le risque imposé}

La question de la répartition des risques et des bienfaits s'accompagne d'une autre interrogation, qui consiste à déterminer si le risque est perçu comme étant supporté délibérément, sous la maîtrise unique de celui qui l'assume. Cet aspect fait également partie du principe interdisant l'imposition de dommages à autrui (voir la section précédente). Aucune personne, réfléchissant l'espace d'un instant à sa propre acceptation du risque, ne s'étonnera de constater la grande différence entre un risque qu'elle accepte délibérément et ce même risque qu'on lui impose. C'est en fait probablement le plus important facteur agissant sur la perception de l'acceptabilité du risque - souvent au-delà même de l'ampleur du risque (Slovic, 1987; Fischhoff, Slovic et Lichtenstein, 2000). Il est évident que les gens qui font du ski, du parachutisme, de l'escalade, etc. ne le font pas parce qu'ils croient que le risque soit très faible. Au contraire, ils savent très bien que les risques sont très élevés. Ces risques sont acceptables principalement parce que la personne les prend délibérément. En fait, on pourrait même dire que ces personnes prennent des risques parce qu'ils aiment le faire, et non pas pour engranger un bienfait compensateur (le plaisir, par exemple).

C'est précisément pour cette raison que certains s'engagent dans des activités que d'autres jugent inacceptables pour eux-mêmes. Si les gens fument, se nourrissent de malbouffe et conduisent des voitures, activités qui comptent toutes parmi les plus risquées de nos jours, ce n'est généralement pas parce qu'ils en ignorent les conséquences (Slovic, 2001; McDaniels et coll., 1992; Fischhoff, Slovic et Lichtenstein, 2000; Read et Combs, 2000). Et pourtant, on voit souvent ces mêmes personnes refuser totalement d'entreprendre d'autres activités beaucoup moins dangereuses, car elles ne veulent pas rajouter des risques supplémentaires à ceux qu'elles supportent déjà, délibérément ou non. C'est ainsi qu'un grand fumeur (risque élevé) insistera pour ne manger que des aliments biologiques cultivés sans recours aux 
pesticides (évitement d'un risque faible) et qu'un adepte de la malbouffe (risque élevé) évitera le parachutisme (risque élevé) et les vols commerciaux (risque très peu élevé). Les experts considèrent souvent ces comportements comme un témoignage de l'irrationalité humaine, quand c'est fréquemment le contraire. Si l'on prend de grands risques en choisissant certaines activités, n'est-il pas logique de réduire d'autres risques afin de circonscrire le fardeau délibérément accepté ${ }^{5}$ ?

C'est parce que l'on perçoit les risques comme étant délibérément choisis qu'on les juge acceptables à première vue. Par contre, si d'autres vous imposent un fardeau de risques plus lourd sans votre consentement, même dans un but tout à fait louable, il est fort peu probable que vous soyez d'accord, même si le nouveau risque demeure très faible (Slovic, 1987, 2001; Thompson, 1986; Sjöberg, 2000). On retrouve ici les considérations d'ordre éthique du principe de non-nuisance. Un des principes fondamentaux de la démocratie libérale veut que les autres n'aient aucun droit de vous imposer des torts, ni même de vous exposer à ce risque. Par conséquent, en règle générale et pour des raisons faciles à comprendre et bien logiques, un risque perçu comme étant imposé, particulièrement au profit de quelqu'un d'autre, sera bien moins acceptable, même si son ampleur réelle (et non pas seulement celle qui est perçue) est relativement faible. Entre un risque délibérément accepté par la personne qui en bénéficie et un risque imposé, il ne peut y avoir de parité. Vous pouvez, par exemple, vous accommoder du risque de griller une cigarette dans un espace qui m'appartient, parce que vous avez choisi de prendre ce risque. Par contre, le risque que vous m'imposez n'est pas acceptable, si faible soit-il, parce que je ne l'ai pas choisi délibérément.

Cette distinction, si évidente et si logique quand on l'examine de plus près, figure parmi celles qui échappent souvent aux experts qui tentent de convaincre des profanes d'accepter différents risques. La stratégie de persuasion qu'ils emploient le plus souvent et qui est aussi la moins efficace est simple et se base sur le « risque comparatif ». En soulignant que le risque associé à une activité imposée (la vaccination, par exemple) est faible comparativement à celui qui découle d'une activité délibérément choisie (être passager dans une voiture, etc.), il est peu probable qu'on arrive à convaincre quiconque de l'acceptabilité du premier. Une meilleure façon de convaincre les gens d'accepter un risque consiste à découvrir les raisons qui risquent de les y inciter (leur dire, par exemple : "Vous pourriez en bénéficier » ou "Vos proches en profiteraient »).

Le phénomène de la réticence à la vaccination est fortement dépendant du facteur de l'acceptation délibérée ou obligée. Quand un programme de vaccination est imposé par un gouvernement, une autorité de la santé, un employeur, une commission scolaire ou toute autre institution puissante, son caractère coercitif apparaît. Si le vaccin en cause ou la vaccination en général inquiètent à cause des risques qu'ils présentent, quel qu'en soit le fondement, ces derniers seront probablement vus comme un fardeau imposé. Si les experts ne peuvent

5. C'est pourquoi il est presque toujours inutile de présenter des arguments comparateurs quand on recherche l'acceptation de risques non consentis. Les promoteurs de technologies perçues comme dangereuses plaident généralement que le risque qu'elles présentent n'est pas plus grand que celui que les gens auxquels ils s'adressent acceptent déjà dans leur vie : « Vous conduisez. II est beaucoup plus probable que vous soyez victime d'un accident mortel que des effets de ma technologie (à cause de la radiation, de l'exposition aux produits chimiques, etc.). » Le raisonnement est le suivant : «Vous acceptez le risque x qui est aussi, sinon plus grand que mon risque y. Vous devriez donc accepter mon risque y. "Évidemment, une personne raisonnable répondra ainsi : "Comment? Vous me dites que je supporte déjà le risque $x$ ? Alors, ça me suffira amplement. Assurément, je ne veux pas doubler mon risque total en y ajoutant votre risque y. » Voilà pourquoi la comparaison des risques est rarement convaincante dans un débat public. 
prétendre avec crédibilité qu'ils n'existent pas, même s'ils sont peu probables, ils seront vus comme étant imposés.

Cette réalité de la perception des risques mène les promoteurs de la vaccination à une double contrainte. Plus grande est la menace que présente la maladie pour la population, plus il faudra que la participation au programme de vaccination soit élargie et, en ce cas, plus forte devra être l'incitation et même l'obligation de participer provenant de la politique publique. En revanche, plus coercitif sera le programme de vaccination, plus la réticence augmentera chez les sous-populations craignant les risques. Ces derniers deviennent moins acceptables, même si on les sait peu importants. Si, dans le contexte social et politique, la perception de coercition est renforcée par une grande méfiance à l'égard des organismes gouvernementaux ou des grands fabricants qui produisent les vaccins, la réticence progressera probablement en conséquence.

Tout cela présente un défi quasi insurmontable en matière de communication, pour les promoteurs de tels programmes. Faire valoir des arguments scientifiques sera rarement convaincant auprès de personnes qui jugent déjà de faibles risques inacceptables en raison d'autres facteurs associés à la perception des risques. Si les scientifiques et les professionnels de santé publique ne peuvent affirmer sans mentir qu'il n'y a aucun risque (ce que les scientifiques spécialistes ne pourront pratiquement jamais établir), il faut prévoir qu'un certain risque demeurera et cela renforce la perception que les experts imposent un risque à toute personne que la vaccination inquiète. Le défi à relever en matière de communication consiste donc à s'attaquer directement au problème. On peut le faire d'abord en aidant les gens à comprendre que les aspects coercitifs du programme sont essentiels pour leur protection ou celle de leurs enfants contre des risques que d'autres leur imposent déjà.

\section{La maîtrise du risque}

L'autonomie est une valeur profondément imbriquée dans la législation et la théorie morale occidentales. La perception d'avoir le contrôle de sa vie a une incidence sur un autre aspect de l'acceptabilité du risque qui s'apparente très étroitement au volontarisme. Un risque qu'une personne a l'impression de contrôler sera jugé acceptable beaucoup plus facilement que dans le cas contraire.

Les gens qui conduisent, par exemple, ont grande confiance en leurs compétences à cet égard, notamment pour éviter les accidents; comme elles ont cette confiance (erronée), le nombre élevé de décès et de blessures dans de tels accidents est attribuable à la moindre compétence des autres chauffeurs et à leur moindre capacité d'éviter les accidents (Slovic, 1987, 2000). Évidemment, le fait que la plupart des gens ne sont pas si compétents ne modifie pas cette perception - ils aiment savoir qu'ils ont la maîtrise des événements et ont une grande confiance en eux. En avion, cependant, les passagers sont à la merci des autres - les pilotes, l'équipage et les mécaniciens ont la maîtrise sur tout. Si les choses tournent mal, les passagers d'un avion, comparativement à ceux d'une voiture, sont entièrement sans recours et ne devront leur salut qu'au personnel ou à Dieu.

Il existe souvent une corrélation entre le facteur du contrôle, dans l'acceptabilité du risque, et le facteur de familiarité décrit précédemment : les risques familiers sont ceux auxquels nous 
nous sommes habitués et pour lesquels nous avons imaginé des mécanismes d'évitement et de contrôle. Cela nous ramène à l'exemple donné dans les passages sur la familiarité, c'està-dire la comparaison entre les maladies relativement familières pour lesquelles des vaccins ont été conçus, comme la rougeole, les oreillons, etc., et les maladies moins connues comme l'autisme. Les maladies peu familières comme l'autisme partagent un aspect important, soit le fait qu'elles échappent presque entièrement à notre contrôle. Nous n'avons aucun moyen d'en prévenir l'apparition et très peu d'indications sur la manière de les contrôler ou d'en limiter les effets dévastateurs. Là encore, peu de gens verront comme équivalentes l'acceptabilité du risque de contracter une maladie incontrôlable comme l'autisme et l'acceptabilité du risque d'attraper une maladie plus gérable, pouvant être prévenue par un vaccin. Il est tout de même paradoxal de constater que les vaccins efficaces sont probablement le meilleur moyen de venir à bout de maladies de ce type. Par conséquent, la crainte très grande générée par le risque négligeable, sinon inexistant qu'un vaccin cause l'autisme trouve en partie son origine dans l'efficacité des vaccins.

\section{Agentivité et responsabilité du risque}

Dans l'évaluation habituelle du risque faite par un expert, un risque est un risque et un dommage est un dommage, quels que soient les sources ou les agents responsables de leur création. Les torts identiques sont également dommageables, quelles que soient leur origine ou les personnes qui en sont responsables. Ce n'est pas ce qu'observent la plupart des profanes et même les experts qui assurent la gestion quotidienne de leurs propres risques. Pour la plupart d'entre nous, la différence est grande si les risques que nous supportons nous sont imposés, d'une part, aléatoirement par la nature ou par le dessein mystérieux d'un dieu ou, d'autre part, par des agents humains raisonnables. La différence est également énorme si nous nous considérons comme le principal agent infligeant des dommages à autrui, particulièrement s'il s'agit de personnes pour lesquelles nous éprouvons la plus grande inquiétude morale et la plus grande responsabilité (notre famille et nos enfants, par exemple). Pour des raisons qu'il est inutile d'expliquer ici (car elles sont profondément imbriquées dans nos valeurs morales et les normes sociales), nous nous attendons à ce que les autres agissent de manière responsable à notre égard et ne nous fassent aucun tort et nous sommes aussi exigeants envers nous-mêmes, dans notre désir de respecter les normes de l'éthique à leur endroit. Les gens sont donc bien moins tolérants quand ils perçoivent qu'un risque leur est imposé par un voisin qu'ils ne le seraient si ce risque ou un risque équivalent leur était imposé par le sort, la nature ou le ou les dieux auxquels ils croient et dont ils acceptent un éventuel dessein sur leur vie.

Par conséquent, de tels risques inéluctables peuvent être moins inquiétants que s'ils provenaient de l'homme, à ampleur égale ou grandement moindre. En d'autres mots, nous nous attendons à ce que nos voisins respectent l'éthique plus que la nature et nous sommes moins tolérants si ce sont eux qui nous imposent le risque. Ce facteur agit dans la réticence à la vaccination que bien des gens fondent sur les convictions religieuses. Dans leur perspective, l'incidence d'une maladie dans une population et la sélection des victimes sont des questions qu'il est impossible d'envisager sans considérer la volonté de Dieu et l'intervention divine dans le monde. C'est pourquoi certains appellent « actes de Dieu » différents événements naturels, y compris les plus tragiques et dévastateurs. II n'est donc pas étonnant que l'on ne considère pas 
les risques d'événements « naturels », comme la maladie, de la même manière que les risques imposés par des agents humains, même chez les gens qui ne se disent pas expressément religieux (Brunk et Hartley, 2012). Les témoignages suivants de personnes interrogées lors d'études sur la réticence à la vaccination témoignent de la plus grande acceptabilité du risque naturel, par rapport au risque anthropique:

Certaines maladies n'ont pas beaucoup d'importance. Être malade se limite souvent à des symptômes; alors pourquoi prendre le risque d'avoir une réaction indésirable [au vaccin]. (Guay et coll., 2014)

Nous disons que la nature fait des merveilles; alors, pourquoi la contredire? Notre environnement nous apporte tout ce qu'il faut; n'y changeons rien. Dans mon cas, quand notre enfant est né, nous pouvions choisir de lui mettre un peu de crème dans les yeux, un petit peu de ci, une petite injection contre ça. Dès la naissance et peutêtre même avant, différentes interventions nous étaient proposées ... Je les ai toutes refusées. (Dubé et coll., 2015)

Pour la plupart d'entre nous, il est particulièrement important de ne pas être l'agent qui cause du tort aux personnes dont nous nous sentons responsables. Nous voyons une grande différence entre l'impression de responsabilité morale que nous ressentons lorsque nous ne causons de tort à personne et celle que nous inspire le fait de prévenir des dommages à autruí. Par conséquent, toutes autres choses étant égales, les risques que les autres nous imposent et ceux que nous nous percevons imposés comme agents nous sont généralement moins acceptables - et nous les tolérons beaucoup moins. Cette acceptabilité moindre découle, dans bien des cas, du fait qu'advenant la concrétisation des dommages - même s'ils sont peu probables et relativement peu importants - on les percevra comme ayant été injustement imposés à autrui par soi-même. Le principe éthique de non-nuisance, si fortement ancré dans l'imagination du public et du secteur des soins de santé, apparaît clairement comme étant l'une des sources de cette perception du risque.

Ce volet de l'acceptabilité du risque intervient fortement dans la perception du risque associé à la vaccination. En dernière analyse, la décision de faire vacciner un enfant se prend par les parents ou le tuteur. Ce consentement est, à tout le moins, un élément indispensable de la vaccination. Si le parent croit ou craint déjà que le vaccin comporte un risque, même négligeable, on ne s'étonnera pas que ses soupçons soient grandement amplifiés s'il sait qu'il impose un tort potentiel à son enfant.

Les sous-cultures qui s'opposent à la vaccination ou qui y sont réticentes s'abreuvent des histoires relatées sur Internet et les réseaux sociaux, où des parents racontent avoir vu leur enfant affligé d'une maladie jusqu'alors inconnue ou non diagnostiquée dans un espace temps relativement rapproché de la vaccination. D'un point de vue scientifique, ce ne sont que des anecdotes, mais elles ont un grand pouvoir sur les parents qui les lisent (Guay et coll., 2014). Ce pouvoir leur vient en grande partie d'une assimilation naturelle à la situation des

6. Les théoriciens de la morale se demandent depuis des siècles si ce point de vue est moralement valable. C'est le débat fondamental entre les théories morales ${ }^{\circ}$ conséquentialistes ${ }^{\circ}$ " (fondées sur l'utilité) et « ${ }^{\circ}$ non conséquentialistes ${ }^{\circ} »$ (fondées sur les règles ou la vertu). Les partisans du non-conséquentialisme estiment que les individus sont dans l'ensemble plus responsables d'assurer l'authenticité morale de leurs gestes que de prévenir les effets des gestes d'autrui. Il est difficile d'établir lequel de ces deux points de vue opposés est le plus valable sur le plan philosophique, mais il semble clair que les sentiments dominants, dans la plupart des cultures (comme le montre notre analyse de la perception des risques), sont favorables au second, sous bien des aspects. 
parents bouleversés à l'idée d'être à l'origine de la souffrance de leur enfant et de l'empathie que l'on éprouve à leur endroit.

Le témoignage chargé d'émotion de la mère épidémiologiste, résumé au début de ce chapitre, est remarquable en ce qu'il exprime bien ce refus de jouer le rôle d'agent moralement responsable du tort subi par l'enfant. On peut supposer que la mère, en tant que scientifique, comprenait la différence entre les preuves scientifiques du risque que présentent les maladies contre lesquelles elle a fait vacciner son enfant et le manque de données probantes montrant que le vaccin a haussé le risque d'autisme dont l'enfant a été plus tard victime. Selon toute probabilité, elle savait également fort bien que le vaccin réduirait de beaucoup la possibilité que son enfant soit atteint des maladies visées par la vaccination. Malgré tout, ce qu'elle dit être l'aspect le plus important de sa propre évaluation du risque n'a aucun lien avec ces probabilités; il réside plutôt dans la possibilité que son choix puisse être à l'origine du tort causé à son enfant, si peu probable soit-elle. Elle avait compris que l'apparition de la maladie chez l'enfant - survenue très peu de temps après la vaccination - n'est qu'un seul point de données et n'a, dès lors, aucune signification scientifique. Dans une discussion avec elle lors de notre rencontre, un autre participant a fait remarquer que son expérience n'est qu'une "observation empirique ». Cependant, en sa qualité de scientifique, elle devrait aussi savoir que de nouvelles preuves peuvent toujours entraîner une révision du consensus et que les «observations empiriques » d'aujourd'hui se transforment parfois en ensembles de données qui font la science de demain.

\section{La vulnérabilité des personnes assujetties au risque}

De l'analyse qui précède ressort une autre facteur influençant l'acceptabilité du risque, selon les études qui portent sur la perception de celui-ci (Raithatha et coll., 2003; Boholm, 1998; Slovic, 1987; Pligt, 1998); ces dernières ont constamment souligné que les risques auxquels sont assujettis les enfants, les personnes âgées et les autres individus vulnérables qui sont incapables de se protéger ou de consentir volontairement à les supporter sont envisagés de manière très différente par la plupart d'entre nous et particulièrement par ceux qui se sentent responsables de ces personnes. Le risque fait naître plus d'inquiétude encore s'il menace des enfants ou d'autres personnes vulnérables que si seulement soi-même ou d'autres individus similaires étaient touchés. Par voie de conséquence, le degré de risque que la plupart des gens estiment acceptable, quand il s'agit d'un aliment ou d'un produit thérapeutique destiné principalement aux adultes, le sera probablement moins s'il est conçu pour les très jeunes enfants ou les personnes très âgées.

De toute évidence, l'inquiétude que l'on éprouve pour une population vulnérable (les enfants) devient un facteur très important chez les réticents à la vaccination, simplement parce que les enfants sont les plus à risque de contracter une maladie évitable par la vaccination; c'est pourquoi la plupart des programmes de vaccination visent la population enfantine. Les précautions que la plupart d'entre nous sommes enclins à prendre à l'égard des enfants et des personnes vulnérables auront pour effet de renforcer les autres facteurs qui entrent en jeu dans la réticence à la vaccination. 


\section{La confiance}

D'une étude à l'autre, le facteur le plus déterminant, en ce qui concerne l'acceptabilité du risque, est la confiance (Slovic, 1987, 2000; Wynne, 2006; Frewer et Miles, 2001; Wildavsky et Dake, 1990; Graffeo et coll., 2004). II est plus probable que nous acceptions un risque, même considérablement plus élevé, si nous avons grande confiance en son créateur ou son gestionnaire; plus la confiance est grande, plus la tendance à l'acceptation sera élevée. Les gens sont prêts à subir des interventions à risque élevé comme des greffes non seulement parce que les autres traitements possibles sont désagréables ou pires encore, mais aussi, en partie, parce que le professionnel et sa profession leur inspirent confiance. En cas d'erreurs ou de fautes professionnelles graves, la relation s'érode et la tendance à l'acceptabilité se modifie radicalement (voir Bramadat au sujet de la "crise de confiance», au premier chapitre).

Quand il est question de l'acceptabilité du risque, l'opposition et la réticence à la vaccination varient en fonction de la confiance de plusieurs manières facilement identifiables. De nombreux observateurs ont noté qu'au sein de la sous-culture réticente, l'ampleur de la méfiance à l'égard du secteur pharmaceutique (à savoir les concepteurs et les fabricants de vaccins) est plus élevée que dans la population en général (McMurray et coll., 2004; se reporter au premier et au quatrième chapitres, sous la plume respective de Bramadat et Roy). La méfiance se nourrit de plusieurs éléments et notamment de cas hautement médiatisés et bien documentés montrant le secteur introduisant dans le marché, avec une vigoureuse promotion, des produits thérapeutiques ayant, comme le Vioxx, causé de graves effets indésirables à ceux qui les ont utilisés. Le secteur a clamé que ces médicaments avaient subi des essais exhaustifs et qu'ils ne présentaient aucun risque inacceptable, mais cela a eu pour résultat d'entraîner à la baisse la confiance accordée à sa compétence et son intégrité. Dès que la confiance dont jouit un gestionnaire de risque est diminuée, il devient très difficile de la restaurer et très peu probable que réussissent des tentatives futures de convaincre les gens de l'innocuité de ces produits, si faibles que soient les risques inhérents réels?.

Àcette méfiance à l'égard des grands fabricants de médicamentss'en ajoute une autre, cette fois dirigée vers les autres intervenants des programmes de vaccination obligatoires. II s'agit notamment des organismes gouvernementaux qui les appliquent et en font la promotion, que les personnes idéologiquement opposées à l'autoritarisme étatique accusent souvent de restreindre les libertés individuelles. Les groupes réticents à la vaccination se méfient aussi de la profession médicale, qu'ils accusent de «s'acoquiner » avec les grands fabricants. La méfiance s'étend facilement à l'ensemble des intervenants, y compris les médecins et les responsables de santé publique, de sorte que la non-acceptabilité du risque est amplifiée, au détriment de l'incitation à la vaccination. La méfiance à l'endroit des intervenants transparaît dans le témoignage d'un sujet mentionné dans l'étude de Guay et coll. (2014) : " [Ma] réticence est basée sur la nécessité. Les études et les recommandations du gouvernement ne m'inspirent pas confiance [à cause] des fabricants de médicaments, que l'intérêt financier incite à vendre leurs vaccins»

7. Pour une relation complète de ce qui s'est produit dans le cas des implants mammaires en silicone de Dow Corning, se reporter à Brunk (1997). 


\section{Fondements éthiques de l'activité productrice de risque}

Parmi les autres facteurs ayant une incidence sur l'acceptabilité du risque, l'un se rapporte à la manière dont la production du risque est perçue dans le système de valeur de l'individu. En d'autres mots, le risque sera moins acceptable si l'activité donnant lieu au tort potentiel est perçue comme contraire à l'éthique par la personne qui le subit.

Au nombre des sources de l'opposition à la vaccination figurent les groupes religieux et leurs membres, qui sont motivés par de fortes interdictions d'ordre moral à l'endroit de la vaccination. Bien que la quasi-totalité de ces individus et de ces groupes soit favorable à la vaccination, un petit nombre affiche des convictions si bien ancrées qu'il est pratiquement impossible pour eux de modifier leurs perspectives. Dans leur cas (voir le premier chapitre de Bramadat et l'annexe du présent ouvrage), l'inquiétude ne vient pas d'une analyse des risques pour la santé présentés par la vaccination; il s'agit plutôt d'un risque moral associé, par exemple, à la perception que le vaccin contre le VPH puisse entraîner l'activité sexuelle chez les adolescents ou la standardiser ou d'une culpabilité morale associée à l'acceptation de vaccins fabriqués au moyen de lignées cellulaires provenant de fœtus avortés. On considère ce risque moral comme inacceptable, mais, en pratique, les personnes qui affichent cette perspective principalement d'ordre moral la renforcent souvent en désignant aussi les risques pour la santé. L'objection au risque moral que présente la vaccination peut trouver son origine dans différentes perspectives théologiques et morales ( $v$. Bramadat, au premier chapitre), y compris celle voulant que la vaccination nuit au dessein de Dieu sur la vie des hommes, ou encore une autre selon laquelle la production de vaccins a recours à des pratiques contraires à l'éthique, comme l'utilisation de lignées cellulaires provenant de fotus avortés.

Il est intéressant de noter cependant que les personnes ayant ces opinions trouvent un moyen de se soumettre à la vaccination obligatoire (ou à d'autres interventions thérapeutiques comme la transfusion de sang chez un enfant) en prétendant que c'est «à leur corps défendant » que les autorités ont pu imposer leur façon de voir. Elles se justifient en disant que le tort ne vient pas d'elles-mêmes, mais d'autrui, et qu'il leur a été imposé contre leur volonté. Dans leur manière de comprendre l'éthique - qui ne se différencie pas beaucoup de l'« agentivité » dont nous avons discuté précédemment - on n'a pas de reproches moraux à leur adresser pour la vaccination (ou la transfusion sanguine), parce que quelqu'un d'autre les y a obligées. C'est alors que le risque moral devient acceptable. Cet argument est notamment devenu l'argument juridique standard aux États-Unis et au Canada, quand il y a un conflit entre l'État et les Témoins de Jéhovah au sujet de transfusions forcées sur les enfants (Singelenberg, 1990).

\section{Le problème de la communication en présence d'une réticence à la vaccination}

Après avoir étudié les facteurs qui entrent en jeu dans une décision d'accepter un risque délibérément ou par obligation, nous avons démontré que l'attitude des individus ou des groupes vis-à-vis d'un risque donné est le résultat de l'application d'un ensemble très complexe de forces sociales, culturelles, religieuses et personnelles. Il devrait être clair que, pour obtenir 
une réponse à la question de savoir si un vaccin (ou une autre technologie) est sans danger, il faut aller beaucoup plus loin que ce que la science permet d'établir. Par conséquent, il serait trop simple d'affirmer que le principal problème à résoudre, dans le débat sur la vaccination, se situe à l'échelle de l'analphabétisme scientifique. On peut dire avec certitude qu'une bonne partie de l'opposition à la vaccination provient d'un refus d'adhérer au consensus scientifique entourant les risques de cette dernière. On peut aussi retenir le rejet de la science et la méfiance qu'elle inspire et qui est renforcée par des perceptions entourant l'acceptabilité des risques même les plu négligeables.

Que doivent donc faire les professionnels de la santé et les autorités en santé publique, qui sont passionnément convaincus de l'importance de la vaccination pour protéger les populations de maladies dangereuses?

Une partie de la réponse réside dans la définition que nous donnons au mot " efficace » dans ce contexte. Si les professionnels de la santé croient que leur principale tâche est de convaincre les réticents d'abandonner leurs convictions et d'adopter la vaccination pour euxmêmes et pour leurs personnes à charge, j'estime que peu de mesures pourront se révéler très efficaces, y compris les campagnes de marketing des sociétés pharmaceutiques. La plupart des chercheurs qui se sont intéressés à la communication des risques (Sandman, 1987; Otway et Wynne, 1989; Pidgeon, Kasperson et Slovic, 2003) ont insisté sur le fait qu'une communication efficace est rarement un processus unidirectionnel allant de l'expert qui persuade le profane - et qu'il ne faut surtout pas se contenter d'ajouter plus de faits et de motifs de les accepter. La communication serait probablement plus efficace si elle prenait la forme d'un dialogue entre l'expert et le profane. Comme François Boucher l'explique au onzième chapitre, le processus ne réussira que si les experts (et particulièrement le personnel clinique de première ligne) portent plus d'attention à tous les facteurs qui constituent les perceptions, des deux côtés, et en apprécient la pertinence.

Une conversation entre des professionnels de la santé et des réticents à la vaccination doit commencer par une description explicite des volets sous-jacents de la perception des risques, de la part des deux parties. Le conflit, en effet, n'est pas uniquement au sujet de la science. Comme j'ai essayé de vous le présenter, il a trait autant à une responsabilité particulière pour les personnes vulnérables, au devoir des parents, à la confiance qu'inspirent la science et les institutions qui y ont recours pour justifier leurs politiques, au choix délibéré et à la maîtrise du risque et à la répartition des risques et des bienfaits qu'à l'influence des convictions religieuses et culturelles, et ainsi de suite.

Le problème réside dans le fait que, contrairement à la science, il n'existe dans notre société aucun consensus sur les valeurs sous-jacentes qui devraient entrer dans l'évaluation des aspects non scientifiques de l'acceptabilité des risques. Non seulement n'y a-t-il pas de consensus, mais il n'existe pas non plus de convention sur ce qui est un moyen « rationnel » de régler les différends au sujet de ces valeurs sous-jacentes. Dans le premier chapitre du présent ouvrage, Paul Bramadat cerne la « crise » qui touche la vérité et en fait une des plus importantes tendances culturelles de notre société, car elle influencerait les attitudes à l'égard des pratiques de soins de santé, y compris la vaccination. Bramadat a raison de voir, dans cette crise, un scepticisme vis-à-vis de toutes des formes de connaissances, y compris la science. Par contre, la plupart de nos semblables qui croient à la préséance de la science et de la médecine fondée sur des données probantes sur le plan rationnel estiment également que la connaissance peut s'acquérir de diverses manières quand il est question de valeurs, d'éthique, 
de religion, d'esthétique et d'autres domaines non scientifiques. Ces questions font l'objet de discussions régulières dans les médias, mais nous le faisons habituellement en recherchant une représentation équitable des divers points de vue et en laissant à l'auditoire la liberté de choisir par lui-même, puisque nous ne pouvons convenir d'une seule réponse exacte.

C'est pourquoi les autorités publiques, les professionnels de la santé et les autres responsables de santé publique sont attirés par le concept idéal de la prise de décision basée sur la science, quand il est question de risque pour la santé. Ces professionnels ont été formés à résoudre des conflits qui peuvent être réduits à des données scientifiques et ils pourraient difficilement s'imaginer autrement. Dans ce monde scientifique, on emploie un vocabulaire commun, un même jeu d'hypothèses et des critères de jugement identiques. C'est pourquoi il est si attrayant, pour les experts du risque, de réduire toutes les situations à un conflit scientifique, malgré le fait évident que bien des aspects de la gestion du risque ne soient pas de cette nature. En fait, la plupart des facteurs entrant en jeu dans l'acceptabilité du risque sont essentiellement une question de valeurs - et non pas de science.

Dans ce cas et si ces conflits ne sont pas soumis aux mêmes approches méthodologiques convenues, ya-t-il un moyen de les résoudre? Premièrement, il est essentiel de ne pas dissimuler ces facteurs relatifs aux valeurs dans la discussion publique, comme on le fait régulièrement. En posant comme hypothèse de départ que les questions sur l'innocuité de la vaccination et sur la place de celle-ci parmi les meilleurs moyens de répondre aux menaces sur la santé individuelle et publique ne relèvent que de la science, nous ne réussirons pas à mettre au jour bon nombre des enjeux importants qui se cachent derrière des opinions conflictuelles. Nous pourrions même négliger quelques-uns des plus importants facteurs qui forment l'opinion des gens sur ces sujets. Si nous demeurons convaincus que ceux qui rejettent le consensus majoritaire sur la valeur de la vaccination ne comprennent tout simplement pas et ne peuvent apprécier les données scientifiques sur son efficacité (et sur les risques d'effets indésirables) et que la solution à ce problème consiste à étendre et améliorer la formation scientifique, il est fort probable que nous ne ferons guère de progrès dans l'apaisement de la controverse. Bon nombre des enjeux réels passeront encore inaperçus. Admettons que tout ce débat est encore caractérisé par une certaine ignorance de la science et que la formation scientifique est importante. Mais cela ne doit pas nous empêcher d'examiner les autres questions à régler. Celles-ci doivent être exposées avec transparence.

Deuxièmement, une fois qu'on aura convenu de l'importance des facteurs non scientifiques et de la nécessité de les étudier, nous devons trouver un moyen de le faire de manière constructive. Est-il possible d'aller au-delà de l'opinion assez répandue dans la société à l'effet que, puisque ce sont des questions de valeurs, il faut toujours les subordonner aux points de vue individuels, subjectifs, ou aux dogmes religieux ésotériques et, par voie de conséquence, qu'il est impossible d'en discuter ou d'y répondre de manière raisonnable? Je ne veux pas alléguer que ces questions peuvent être traitées de la même façon que celles qui sont de nature scientifique ou empirique. Cependant, comme nous voulons le faire valoir dans cet ouvrage, il est possible d'en débattre de manière rationnelle et courtoise, d'en tirer des enseignements réciproques et de modifier nos opinions entre-temps au besoin, parfois en reconnaissant que la perspective de l'autre mérite le respect. Il y a moyen de poursuivre ces délibérations raisonnables dans des tribunes publiques, dans les médias et dans des séances de counseling privées, où le professionnel et le patient cherchent à en arriver à une juste décision sur la santé de celui-ci, celle de ses personnes à charge ou la santé publique en général. Pour que 
cela se produise de manière fructueuse, cependant, les experts et les profanes intéressés doivent être bien informés, non seulement sur les questions scientifiques cruciales, mais aussi sur les enjeux critiques en matière de valeurs, y compris celles qui sont profondément enracinées dans des convictions religieuses, des textes, des habitudes et des collectivités ou qui se développent sous leur influence. Il est possible de discuter de ces dernières questions dans le respect et la rationalité, en apprenant d'un côté et en persuadant de l'autre. Ce sera la meilleure façon de progresser dans les débats sur la réticence à la vaccination. 


\section{BIBLIOGRAPHIE}

Boholm, A. 1998. "Comparative Studies of Risk Perception: A Review of Twenty Years of Research." Journal of Risk Research 1 (2): 135-63. http://dx.doi.org/10.1080/ 136698798377231.

Brunk, C. 1997. "Silicone Breasts." in Mad Cows and Mother's Milk: The Perils of Poor Risk Communication, edited by D. Powell and W. Leiss, 99-120. Montreal: McGill-Queen's University Press.

Brunk, C., and S. Hartley. 2012. Designer Animals: Mapping the Issues in Animal Biotechnology. Toronto: University of Toronto Press.

Brunk, C., L. Haworth, and B. Lee. 1991. Value Assumptions in Risk Assessment. Waterloo, ON: Wilfrid Laurier University Press.

Dubé, E., M. Vivion, C. Sauvageau, A. Gagneur, R. Gagnon, and M. Guay. 2015. "'Nature Does Things Well, Why Should We Interfere?': Vaccine Hesitancy Among Mothers." Qualitative Health Research 26 (3): 411-25. http://dx.doi.org/10.1177/1049732315573207.

Editors of The Lancet. 2010. "Retraction - Ileal-Lymphoid-Nodular Hyperplasia, Non- Specific Colitis, and Pervasive Developmental Disorder in Children." The Lancet 375 (9713): 445. http://dx.doi.org/10.1016/ S0140-6736(10)60175-4.

Fischhoff, B. 1998. "Risk Perception and Communication Unplugged: Twenty Years of Process." in The Earthscan Reader in Risk and Modern Society, edited by R. Lofstedt and L. Frewer, 133-45. London: Earthscan.

Fischhoff, B., P. Slovic, and S. Lichtenstein. 2000. "Weighing the Risks: Which Risks Are Acceptable?" in The Perception of Risk, edited by P. Slovik, 121-36. London: Earthscan.

Fischhoff, B., P. Slovic, and S. Lichtenstein, S. Read and B. Combs. 2000. "How Safe is Safe Enough? A Psychometric Study of Attitudes toward Technological Risks and Benefits." in The Perception of Risk, edited by P. Slovik, 80-103. London: Earthscan.

Frewer, L.J., and S. Miles. 2001. "Risk Perception, Communication and Trust. How Might Consumer Confidence in the Food Supply be Maintained?" in Food, People and Society: A European Perspective of Consumers' Food Choices, edited by L. Frewer, E. Risvik, and H. Schifferstein, 401-13. Berlin: Springer. http://dx.doi. org/10.1007/978-3-662-04601-2_24.

Frewer, L.J., R. Shepherd, and P. Sparks. 1994. "Biotechnology and Food Production: Knowledge and Perceived Risk." British Food Journal 96 (9): 26-32. http://dx.doi. org/10.1108/00070709410072562.

Graffeo, M., L. Savadori, L. Lombardi, K. Tentori, N. Bonini, and R. Rumiati. 2004. "Trust and Attitude in Consumer Food Choices under Risk." Agrarwirtschaft 53 (8): 319-27.

Guay, M., M. Ghorbel, J. Lemaire, E. Cadieux, J. Désilets, P. Clément, C. Vanier, S. Briand, E. Dubé, C. Boulet, et al. 2014. "Vaccine Hesitation among Quebec Parents of Children Aged 2 Months to 5 Years." Poster presented at the Canadian Immunization Conference, Ottawa, ON, 2-4 December.

Kahneman, D. 2011. Thinking Fast and Slow. New York: Farrar, Strauss \& Giroux.

Kahneman, D., P. Slovic, and A. Tversky, eds. 1982. Judgment under Uncertainty: Heuristics and Biases. New York: Cambridge University Press. http://dx.doi. org/10.1017/CBo9780511809477.

McDaniels, T.L., M.S. Kamlet, and G.W. Fischer. 1992. "Risk Perception and the Value of Safety." Risk Analysis 12 (4): 495-503. http://dx.doi.org/10.1111/j.1539-6924.1992.tb00706.x.

McMurray, R., F.M. Cheater, A. Weighall, C. Nelson, M. Schweiger, and S. Mukherjee. 2004. "Managing Controversy through Consultation: A Qualitative Study of Communication and Trust around MMR Vaccination Decisions." British Journal of General Practice 54 (504): 520-5. 
Mill, J.S. 1859. De la liberté. London: Longman, Roberts \& Green.

Otway, H., and B. Wynne. 1989. "Risk Communication: Paradigm and Paradox." Risk Analysis 9 (2): 141-5. http://dx.doi.org/10.1111/j.1539-6924.1989.tb01232.x.

Petts, J., and S. Niemeyer. 2004. "Health Risk Communication and Amplification: Learning from the MMR Vaccination Controversy." Health Risk \& Society 6 (1): 7-23. http://dx.doi.org/10.1080/136985704100016 78284.

Pidgeon, N., R.E. Kasperson, and P. Slovic, eds. 2003. The Social Amplification of Risk. Cambridge: Cambridge University Press. http://dx.doi.org/10.1017/CBo9780511550461.

Pligt, J. 1998. "Perceived Risk and Vulnerability as Predictors of Precautionary Behaviour." British Journal of Health Psychology 3 (1): 1-14. http://dx.doi.org/10.1111/j.2044-8287.1998.tb00551.x.

Raithatha, N., R. Holland, S. Gerrard, and i. Harvey. 2003. "A Qualitative investigation of Vaccine Risk Perception amongst Parents Who Immunize Their Children: A Matter of Public Health Concern." Journal of Public Health 25 (2): 161-4. http://dx.doi.org/10.1093/pubmed/fdg034.

Rescher, N. 1983. Risk: A Philosophical Introduction to the Theory of Risk Evaluation and Management. Washington, DC: University Press of America.

Sandman, P.M. 1987. "Risk Communication: Facing Public outrage." Environmental Protection Agency Journal 13: 21.

Sen, A. 1985. "Rationality and Uncertainty. Theory and Decision." Journal of the American College of Toxicology 18: 109-27.

Singelenberg, R. 1990. "The Blood Transfusion Taboo of Jehovah's Witnesses: Origin, Development and Function of a Controversial Doctrine." Social Science \& Medicine 31 (4): 515-23. http://dx.doi.org/10.1016/0277-9536(90)90048-W.

Sjöberg, L. 2000. "Factors in Risk Perception." Risk Analysis 20 (1): 1-12. http://dx.doi.org/10.1111/02724332.00001 .

Slovic, P. 1987. "Perception of Risk." Science 236 (4799): 280-5. http://dx.doi.org/10.1126/science.3563507

- 2000. "Perceived Risk, Trust and Democracy." in The Perception of Risk, edited by P. Slovik, 316-26. London: Earthscan.

- ed. 2001. Smoking: Risk, Perception, and Policy. Thousand Oaks, CA: Sage Publications, Inc. http://dx.doi. org/10.4135/9781452232652.

Thompson, P.B. 1986. "The Philosophical Foundations of Risk." Southern Journal of Philosophy 24 (2): 273-86. http://dx.doi.org/10.1111/j.20416962.1986.tb01566.x.

Tillich, P. 1957. Dynamics of Faith. New York: Harper and Row.

Tversky, A., and D. Kahneman. 1974. "Judgment under Uncertainty: Heuristics and Biases." Science 185 (4157): 1124-31. http://dx.doi.org/10.1126/science.185.4157.1124.

von Winterfeldt, D. 1992. "Expert Knowledge and Public Values in Risk Management: The Role of Decision Analysis." in Social Theories of Risk, edited by S. Krimsky and D. Golding, 321-42. London: Praeger.

Wakefield, A.J., S.H. Murch, A. Anthony, J. Linnell, D.M. Casson, M. Malik, M. Berelowitz, A.P. Dhillon, M.A. Thomson, P. Harvey, et al. 1998. "Illeal-Lymphoid- Nodular Hyperplasia, Non-Specific Colitis, and Pervasive Developmental Disorder in Children." The Lancet 351 (9103): 637-41. http://dx.doi.org/10.1016/ S0140-6736(97)11096-0.

Wildavsky, A., and K. Dake. 1990. "Theories of Risk Perception: Who Fears What and Why?" Daedalus 119: 41-60. 
Wynne, B. 2006. "Public Engagement as a Means of Restoring Public Trust in Science - Hitting the notes, but Missing the Music?" Community Genetics 9 (3): 211-20. 